\title{
Estratégias de Artrópodes Contra Predação por Formigas em Floresta Secundária no Rio de Janeiro
}

\author{
Fábio Souto Almeida1, Luciano Martins ${ }^{\bowtie}$ \& Alejandro G. Farji-Brener³
}

1. Universidade Federal Rural do Rio de Janeiro, Programa de Pós-Graduação em Ciências Ambientais e Florestais/ Departamento de Ciências Administrativas e do Ambiente/ ITR, e-mail: fbio almeida@yahoo.com.br. 2. Universidade Federal Rural do Rio de Janeiro,Programa de PósGraduação em Biologia Animal/ Departamento de Biologia Animal/ IB, e-mail: sphex luciano@yahoo.com.br (Autor para correspondência ${ }^{\bowtie}$ ). 3. CRUB-Universidad Nacional del Comahue, INIBIOMA-CONICET, e-mail: alefarji@yahoo.com

\section{EntomoBrasilis 3 (3): 69-72 (2010)}

Resumo. Objetivou-se avaliar a eficiência de estratégias de artrópodes contra a predação por formigas. Realizamos simulações de estratégias antipredatórias para testar as seguintes hipóteses: esconder-se entre os componentes da serapilheira é uma boa estratégia para um artrópode evitar a predação por formigas; a estratégia de alguns artrópodes de manterem-se suspensos por um fio é eficaz contra predação; variações na estratégia de manter-se suspenso afetam sua eficiência; a predação varia com a altura da presa em relação ao solo. Realizamos dois experimentos numa floresta secundária em Angra dos Reis-RJ. No primeiro, a serapilheira foi removida de parcelas e aplicou-se os seguintes tratamentos: exposto (um grão de arroz sobre o solo); uma folha (um grão de arroz entre o solo e uma folha); duas folhas (um grão de arroz entre duas folhas); quatro folhas (um grão de arroz entre quatro folhas). No segundo, utilizou-se os seguintes tratamentos: suspenso - um fio preso em dois galhos de plantas e um grão de arroz fixo na ponta de um segundo fio, criando uma estrutura em forma de "T"; pêndulo - um fio preso a um galho e na outra extremidade um grão de arroz; folha - um grão de arroz sobre uma folha; serapilheira - um grão de arroz sobre a serapilheira. O suspenso e o pêndulo mostraram-se eficazes, mas o suspenso foi mais eficiente. A altura da isca em relação ao solo não influenciou probabilidade de ocorrer a remoção. Esconder-se entre componentes da serapilheira pode ser um mecanismo de defesa eficiente contra a predação por formigas.

Palavras-chave: Defesas; Formicidae; serapilheira

\section{Arthropod Strategies Against Predation by Ants in a Secondary Forest in Rio de Janeiro, Brazil}

Abstract. We assessed the efficiency of arthropod strategies against predation by ants. Simulations of anti-predator strategies were carried out to test the following hypotheses: hiding in the leaf litter is efficient to escape predation by ants; hanging by a thread is also efficient against ants; variations in the strategy of hanging by a threat affect its efficiency; predation depends on the height of the prey from the ground. We carried out two experiments in a second-growth forest in Angra dos Reis, southeastern Brazil. In the first experiment, leaf litter was removed from the plots and we exposed rice grains to four treatments: a rice grain on the ground (exposed); a rice grain between the ground and a leaf (one leaf); a rice grain between two leaves (two leaves); a rice grain between four leaves (four leaves). In the second experiment, we used other four treatments: a thread attached to two branches of a plant and a second thread hanging from it, with a rice grain attached to its extremity, forming a T-shaped structure (hanging); a thread attached to a branch, with a rice grain attached to its other extremity (pendulum); a rice grain on a leaf (leaf); a rice grain on the leaf litter (leaf litter). Hanging was more efficient than pendulum; though these both strategies showed to be efficient. The height of the bait above ground did not affect its removal probability. Hiding in the leaf litter can be considered as an efficient defense mechanism against predation by ants.

Keywords: Defenses; Formicidae; leaf litter

$\mathbf{Q}$ ualquer característica desenvolvida por um animal que reduza a probabilidade de ser atacado e morto por predadores pode ser considerada um mecanismo defensivo (EDMUNDS 1974). Os predadores atuam como agentes seletivos na evolução das suas presas, pois todas desenvolveram defesas de natureza comportamental, física, morfológica ou química contra a predação (JANZEN 1969; BEGON et al. 1990; Dyer 1995). Para que ocorra a predação, um indivíduo deve ser detectado, atacado, capturado, subjugado e consumido pelo predador, sendo que as defesas devem interromper o processo em uma dessas etapas (BEGON et al. 1990). Assim, os mecanismos de defesa podem ser divididos em primários, efetivos antes de o predador iniciar o ataque, e secundários, que aumentam as chances de um indivíduo sobreviver ao encontro com o predador (EDMUNDS 1974). O aposematismo, a camuflagem e o mimetismo são exemplos de mecanismos primários de defesa (Futuyma 2002; GENTRY \& DYER 2002). Já a fuga, direcionar o ataque para uma parte não vulnerável ou retaliar utilizando armas como espinhos ou substâncias repelentes são exemplos de mecanismos secundários de defesa. Esconder-se de predadores é uma das estratégias anti-predatórias mais comuns e pode ser classificada, dependendo da circunstância, tanto como um mecanismo primário quanto secundário (EDMUNDS 1974; BEGON et al. 1990).

As formigas sãoimportantes predadoras, principalmente de outros artrópodes, são muito abundantes e habitam quase todos os ambientes terrestres (CuEZzo 1998; FLOREN et al. 2002; Tillberg \& BREED 2004). Também apresentam estratégias de forrageamento diversificadas (ToBIN 1995), embora a maioria das espécies forrageie na serapilheira (SchutTe et al. 2007). Além disso, as formigas possuem elevada habilidade para perceber odores, o que é útil para encontrar alimentos (HöLLDOBLER \& Wilson 1990). Como consequência, diversos animais que vivem sobre o solo adquiriram tipos de defesa contra a predação por formigas, como secreções repelentes, exoesqueleto resistente e a ação de matar e comer formigas detectoras, evitando o recrutamento (YAMAGUCHI \& HASEGAWA 1996). Também é possível que as presas ao se esconderem em meio a serapilheira possam diminuir as chances de serem encontradas pelas formigas. Contudo, ao se manterem expostas, a probabilidade de serem localizadas seria maior (Отіs et al. 1986).

Por outro lado, diversas espécies de formigas forrageiam sobre plantas, o que levou diversos organismos que vivem na 
vegetação a desenvolverem estratégias contra predação (ToBIN 1991; Coley \& BARONE 1996). Por exemplo, algumas espécies de aranhas e de lepidópteros na fase de lagarta e pupa, se mantém suspensos por fios (SALAZAR \& WHITMAN 2001; LiLL et al. 2007), dificultando a aproximação de predadores. Esse hábito pode se configurar em uma estratégia contra predação por formigas, pois elas teriam dificuldade de caminhar sobre o fio (Jones et al. 2002). Todavia, afastar-se do solo, vivendo sobre plantas, também pode ser um hábito eficiente para que um organismo potencialmente presa de formigas possa diminuir a probabilidade de ser encontrado pelas predadoras, já que a maioria da mirmecofauna habita a serapilheira (SchuTte et al. 2007).

Assim, o trabalho objetivou avaliar a eficiência de estratégias de artrópodes contra a predação por formigas. Foram realizadas simulações de estratégias anti-predatórias para testar as seguintes hipóteses: (1) esconder-se entre os componentes da serapilheira é uma boa estratégia para um artrópode evitar sua predação por formigas; (2) a estratégia de aranhas e de lepidópteros de manterem-se suspensos por um fio é eficaz contra a predação; (3) variações da estratégia de manter-se suspenso afetam sua eficiência; (4) a predação varia com a altura da presa em relação ao solo.

\section{MATERIAL E MÉTODOS}

A coleta de dados foi realizada no mês de novembro de 2009, em uma floresta secundária em avançado estágio de regeneração situada no distrito de Ilha Grande, Angra dos Reis-RJ ( $23^{\circ} 11^{\prime}$ oo"S, $\left.44^{\circ} 11^{\prime} 44^{\prime} W\right)$. Segundo a classificação de Köppen, o clima da região caracteriza-se como Af (tropical úmido), sem estação seca definida e com precipitação anual média de 2.200 mm (Rocha-PEsSÔA \& Rocha 2008).

Com o objetivo de testar as hipóteses, foram realizados dois experimentos, utilizando-se grãos de arroz cozido para simular possíveis presas de formigas, como pupas de insetos. No primeiro experimento, foram escolhidos ao acaso 40 locais distantes no mínimo 10 metros em si. Em cada local foram demarcadas quatro parcelas de $10 \mathrm{~cm} \times 10 \mathrm{~cm}$, separadas $10 \mathrm{~cm}$ entre si. A serapilheira foi removida das parcelas e aplicaram-se tratamentos que visavam simular diferentes níveis de exposição de um organismo presa de formigas, são eles: exposto (um grão de arroz cozido colocado sobre o solo); uma folha (um grão de arroz cozido colocado entre o solo e uma folha); duas folhas (um grão de arroz cozido colocado entre duas folhas); quatro folhas (um grão de arroz cozido colocado entre quatro folhas). As folhas utilizadas no experimento possuíam tamanho (comprimento 8 ,ocm e largura $\cong 3,0 \mathrm{~cm}$ ) e forma parecidos.

No segundo experimento, foram feitas simulações de técnicas contra predação utilizadas por espécies de aranhas e lepidópteros. Foram aplicados os seguintes tratamentos: suspenso - um fio de costura preso em dois galhos de plantas e um grão de arroz cozido fixo na ponta de um segundo fio, criando uma estrutura em forma de "T"; pêndulo - um fio de costura preso a um galho e tendo na outra extremidade um grão de arroz cozido; folha - um grão de arroz cozido colocado sobre uma folha; serapilheira - um grão de arroz cozido colocado sobre a serapilheira, como tratamento controle (Figura 1). Foram realizadas 48 repetições com 10 metros de distância entre cada uma e as simulações foram instaladas entre 30 e $150 \mathrm{~cm}$ de altura em relação ao solo.

Em ambos os experimentos, as iscas permaneceram expostas no campo durante duas horas. Quando as formigas removeram os grãos de arroz foi considerado que haveria predação caso a isca fosse um organismo vivo, sendo que também foi considerada como remoção a coleta de fragmentos das iscas. As formigas que foram observadas removendo as iscas foram coletadas e identificadas ao nível de gênero com base na chave de Bolton (1994).

Foi utilizado o teste de Qui-quadrado $\left(\chi^{2}\right)$ para determinar a eficiência das estratégias anti-predatórias e a regressão logística para analisar a influência da altura em relação ao solo sobre a remoção das iscas, a 5\% de probabilidade (ZAR 1999).
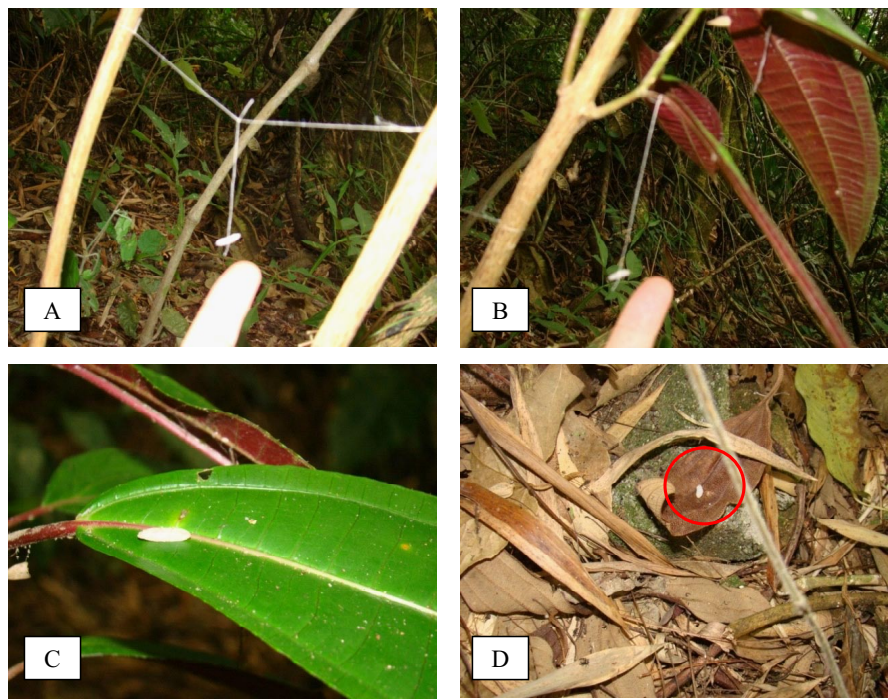

Figura 1. Tratamentos utilizados na avaliação de estratégias contra predação por formigas. A - suspenso; B - pêndulo; C - folha; D serapilheira.

\section{RESULTADOS E DISCUSSÃO}

No primeiro experimento, as formigas que foram coletadas removendo as iscas pertenciam aos gêneros Crematogaster, Odontomachus, Pachycondyla, Pheidole e Solenopsis, sendo que Pheidole foi o único gênero que ocorreu em todos os tratamentos. No geral, a porcentagem de iscas removidas foi de $83,13 \%$. Entre os tratamentos, a remoção aumentou significativamente com o aumento da exposição das iscas $\left(\chi^{2}=18,67, p<0,01\right)$ (Figura 2).

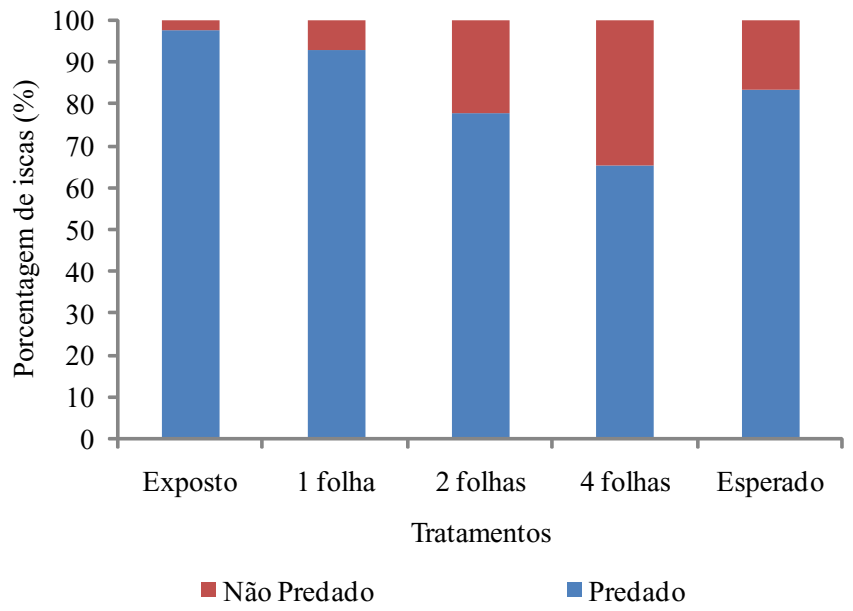

Figura 2. Porcentagem de iscas removidas por formigas em função dos diferentes tratamentos $\left(\chi^{2}=18,67, p<0,01\right)$. Primeiro experimento.

Apesar das formigas perceberem odores com elevada eficiência, foi mais difícil perceber a presença do alimento nas situações em que estava menos exposto. Assim, esconder-se entre os componentes da serapilheira pode ser um mecanismo primário de defesa eficiente contra a predação por formigas. NogueIra-De-SÁ \& Trigo (2005) observaram que indivíduos da espécie Plagiometriona aff. flavescens (Boheman) (Coleoptera: Chrysomelidae) possuem um mecanismo primário de defesa contra formigas, esses organismos protegem-se de predadores quimicamente orientados através da camuflagem química, já que hidrocarbonetos cuticulares das larvas e de folhas de sua planta hospedeira apresentam elevada similaridade. Devido a esta estratégia, foi observado em experimentos de laboratório que formigas da espécie Camponotus crassus (Mayr) não são capazes de encontrar as larvas do coleóptero. Os resultados demonstram 
que determinadas estratégias anti-predação podem superar, ao menos em parte, a habilidade das formigas encontrarem alimento. Todavia, a porcentagem de iscas removidas foi elevada, mesmo no tratamento em que a isca estava menos exposta. Isso confirma a eficiência da mirmecofauna para encontrar presas. É possível que ambientes com maior quantidade e heterogeneidade de serapilheira favoreçam os organismos potencialmente presas de formigas, por fornecerem mais locais para refúgio e dificultar a sua localização. Porém, esse efeito pode ser contrabalanceado, pois ambientes com essas características também podem possuir maior abundância e riqueza de espécies de formigas (VARGAS et al. 2007).

No segundo experimento foram encontrados os gêneros Crematogaster, Odontomachus, Pachycondyla, Pheidole, Pseudomyrmex e Solenopsis (Figura 3). Considerando-se todos os tratamentos, a porcentagem de iscas removidas foi de $43,31 \%$. A porcentagem de iscas removidas foi maior no tratamento denominado serapilheira que sobre as folhas, além disso, o pêndulo e o suspenso mostraram-se estratégias eficazes contra a predação $\left(\chi^{2}=54,8 ; p<0,01\right)$ (Figura 4$)$. Todavia, a porcentagem de iscas removidas foi menor notratamento denominado suspenso que no pêndulo. A remoção não se relacionou significativamente com a altura das iscas quando colocadas sobre folhas $\left(\chi^{2}=2,36\right.$; $\mathrm{p}=0,12)$ ou quando se aplicou a estratégia aqui denominada de pêndulo $\left(\chi^{2}=0,42 ; p=0,51\right)$. Não foi realizada a análise de regressão logística para a estratégia suspenso, pois somente seis das 48 iscas foram removidas.
Os predadores, muitas vezes, exercem expressiva influência sobre as populações das suas presas, sendo que os indivíduos mais adaptados para evitar a predação sobrevivem e se reproduzem. Desse modo, o predador atua como um agente seletivo na evolução das estratégias defensivas da presa (Silva et al. 1997; Azevedo \& Ramalho 1999). Como a estratégia de permanecer suspenso por um fio mostrou-se eficiente contra a aproximação de formigas, é provável que a ação da mirmecofauna sobre as aranhas e lepidópteros tenha sido fundamental para o surgimento dessa adaptação contra predação. Também é provável que a maior eficiência do tratamento denominado suspenso deva-se a sua maior complexidade estrutural. Pode-se dizer que o suspenso impõe ao predador maior dificuldade para alcançar a presa quando comparado com o pêndulo, pois possui um fio na posição horizontal e outro na vertical.

Como houve maior remoção das iscas colocadas sobre a serapilheira que sobre as folhas, pode-se supor que a probabilidade de um organismo ser encontrado por formigas nas plantas é menor que na serapilheira. Entretanto, apesar da maioria das espécies de formigas forragearem na serapilheira, a altura em que as iscas foram expostas nas plantas não influenciou a remoção. O intervalo de variação da altura $(30-150 \mathrm{~cm})$ pode não ter sido suficiente para constatar a influência da altura da isca em relação ao solo sobre a remoção. Em experimentos futuros sugere-se que a variação da altura de exposição da isca seja maior.
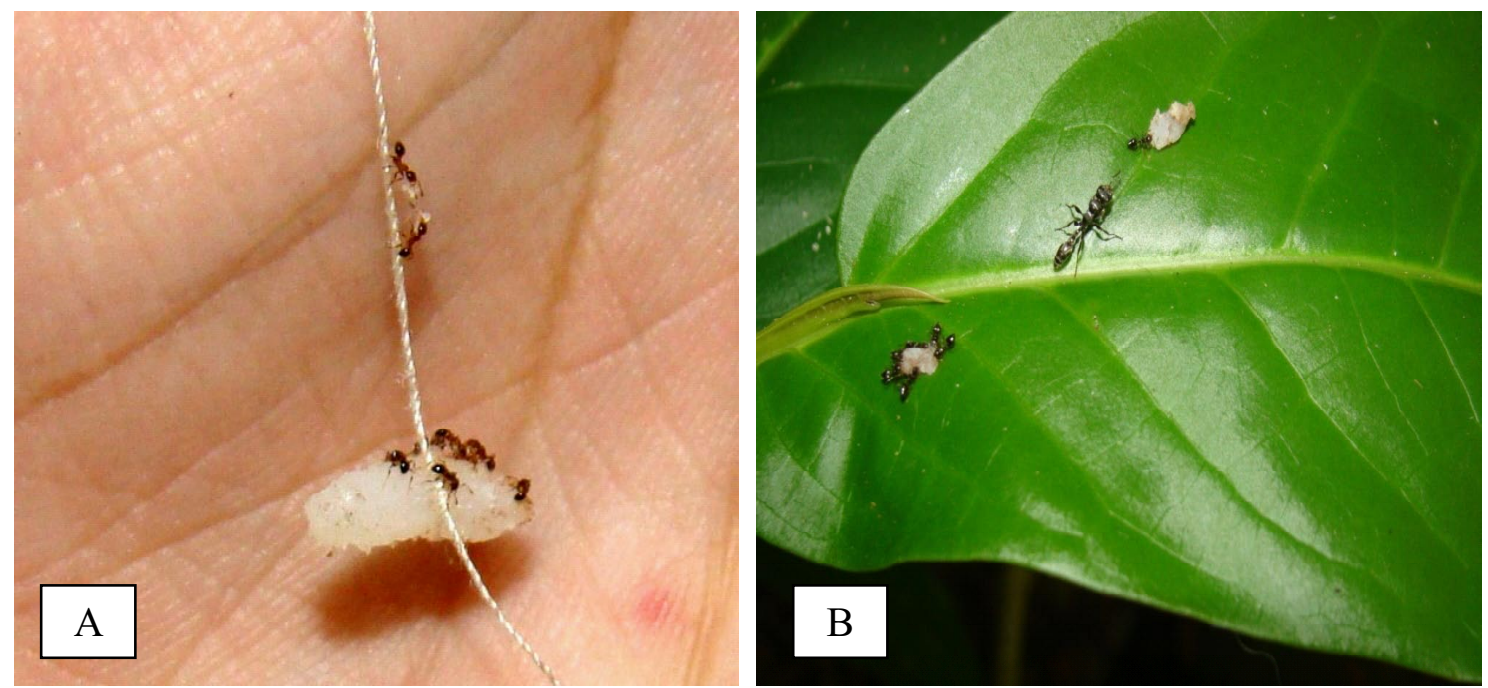

Figura 3. Formigas do gênero Pheidole removendo fragmentos de uma isca no tratamento pêndulo (A) e do gênero Crematogaster e Pseudomyrmex removendo uma isca na folha (B).

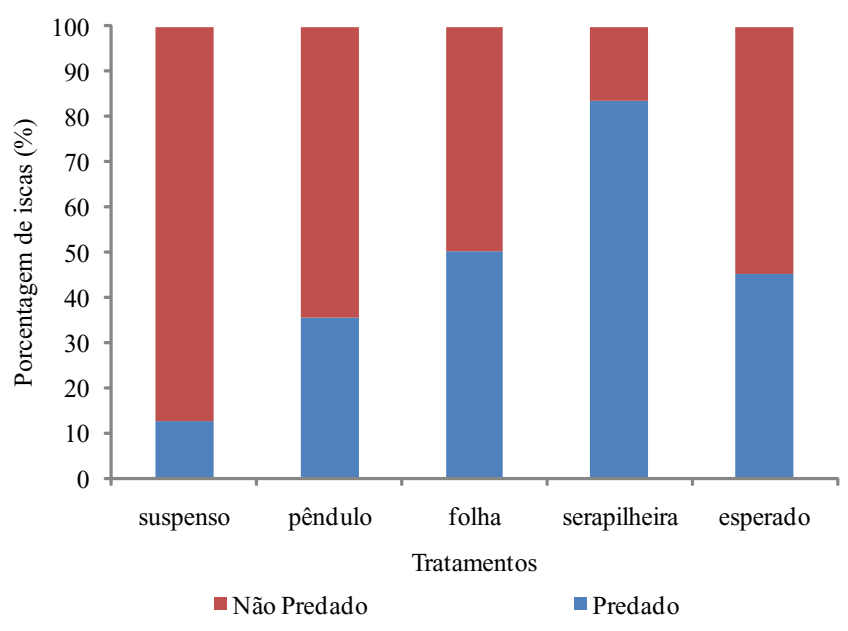

Figura 4. Porcentagem de iscas removidas por formigas em função dos diferentes tratamentos $\left(\chi^{2}=54,8 ; p<0,01\right)$. Segundo experimento.
Os resultados demonstram que, para pequenos artrópodes, esconder-se entre os componentes da serapilheira pode ser um mecanismo de defesa eficiente contra a predação por formigas. Demonstram também que a estratégia de se manter suspenso por um fio, utilizada por algumas espécies de aranhas e de lepidópteros, é eficaz para diminuir a predação por formigas e a complexidade da estrutura de fios influencia a sua eficiência. Para as iscas colocadas sobre plantas, a altura em que foram expostas em relação ao solo não afetou a probabilidade de ocorrer a remoção.

\section{AGRADECIMENTOS}

Agradecemos a Coordenação de Aperfeiçoamento de Pessoal de Nível Superior (CAPES) pelas bolsas de estudo concedidas aos dois primeiros autores. Agradecemos também a Antônio José Mayhé-Nunes e Jarbas Marçal de Queiroz, professores da disciplina de Ecologia de Formigas da UFRRJ, durante a qual os dados do presente experimento foram coletados. A UERJ pela estadia em sua base em Ilha Grande. Agradecemos também aos revisores anônimos pelas críticas e sugestões. 


\section{REFERÊNCIAS}

Azevedo, F.R. \& F.S. Ramalho, 1999. Efeitos da temperatura e da defesa da presa no consumo pelo predador Supputius cincticeps (Stäl) (Heteroptera: Pentatomidae). Pesquisa Agropecuário Brasileira, 34: 165-171.

Begon, M., J.L. Harper \& C.R. Townsend, 1990. Ecology: individuals, populations and communities. Tauton, Blackwell Science, 945p.

Bolton, B., 1994. Identification Guide to the Ant Genera of the World. Massachusetts, Harvard University Press, 222p.

Coley, P.D. \& J.A. Barone, 1996. Herbivory and plant defenses in tropical forests. Annual Review Ecology and Systematics, 27: 305-335.

Cuezzo, F., 1998. Formicidae, p.452-462. In: Morrone, J.J. \& S. Coscarón. Biodiversidad de artropodos argentinos. La Plata, Ediciones SUR, $599 \mathrm{p}$.

Dyer, L.A., 1995. Tasty generalists and nasty specialists? Antipredator mechanisms in tropical lepidopteran larvae. Ecology, 76:1483-1496.

Edmunds, M., 1974. Defense in animals: a survey of antipredator defenses. Harlow, Longman Group Limited, 357 p.

Floren, A., A. Biun \& K.E. Linsenmair, 2002. Arboreal ants as key predators in tropical lowland forest trees. Oecologia, 131: 137-144.

Futuyma, D.J., 2002. Biologia Evolutiva. Ribeirão Preto, FUNPEC, 631p.

Gentry, G.L. \& L.A. Dyer, 2002. On the conditional nature of neotropical caterpillar defenses against their natural enemies. Ecology, 83: 11.

Hölldobler, B. \& E.O. Wilson, 1990. The ants. Cambridge, Belknap/Harvard University Press, 732p.

Janzen D.H., 1969. Seed-eaters versus seed size, number, toxicity and dispersal. Evolution, 23:1-27.

Jones, M.T., I. Castellanos \& M.R. Weiss, 2002. Do leaf shelters always protect caterpillar from invertebrate predators? Ecological Entomology, 27: 753-757.

Lill, J.T., R.J. Marquis, M.A. Walker \& L. Peterson, 2007. Ecological consequences of shelter sharing by leaftying caterpillars. Entomologia Experimentalis et Aplicata, 124: 45-53.

Nogueira-de-Sá, F. \& J.R. Trigo, 2005. Faecal shield of the tortoise beetle Plagiometriona aff. flavescens (Chrysomelidae: Cassidinae) as chemically mediated defence against predators. Journal of Tropical Ecology, 21: 189-194.

\section{Como citar este artigo:}

Almeida, F.S., L. Martins \& A.G. Farji-Brener, 2010. Estratégias de Artrópodes Contra Predação por Formigas em Floresta Secundária no Rio de Janeiro. EntomoBrasilis, 3(3): 69-72. www.periodico.ebras.bio.br/ojs
Otis, G.W., E.C. Santana, D.L. Crawford \& M.L. Higgins, 1986. The effect of foraging army ants on leaf-litter arthropods. Biotropica, 18: 56-61.

Rocha-Pessôa, T.C. \& C.F.D. Rocha, 2008. Reproductive cycle of Pitcairnia Flammea Lindl. (Bromeliaceae/ Pitcairnioideae) in an insular Atlantic rainforest area in southeastern Brazil. Flora, 203: 229-233.

Salazar, B.A. \& D.W. Whitman, 2001. Defensive tactics of caterpillars against predators and parasitoids, p.161-207. In: Ananthakrishnan, T.N. (Ed). Insects and plants defenses dynamics. Plymouth, Science Publishers, 253 p.

Schutte, M.S., J.M. Queiroz, A.J. Mayhe-Nunes \& M.P.S. Pereira, 2007. Inventário estruturado de formigas (Hymenoptera, Formicidae) em floresta ombrófila de encosta na ilha da Marambaia, RJ. Iheringia, Série Zoologia, 97: 103-110.

Silva, E.N., T.M. Santo \& F.S. Ramalho, 1997. Consumo alimentar e crescimento do predador Supputius cincticeps (Stäl) (Heteroptera:Pentatomidae) alimentando-se de lagartas de curuquerê-do-algodoeiro. Anais da Sociedade de Entomologia do Brasil, 26: 349-357.

Tillberg, C.V. \& M.D. Breed, 2004. Placing an omnivore in a complex food web: dietary contributions to adult biomass of an ant. Biotropica, 36: 266-271.

Tobin, J.E., 1991. A neotropical rainforest canopy ant community: some ecological considerations, p. 536-538. In: Huxley C.R. \& D.F. Cutler (Eds). Ant-Plant Interactions. Oxford, Oxford University Press, $601 \mathrm{p}$.

Tobin, J. E., 1995. Ecology and diversity of tropical forest canopy ants, p. 129-147. In: Lowmam, M.D. \& N.M. Nadkarni. Forest canopies. San Diego, Academic Press, 624 p.

Vargas, A.B., A.J. Mayhé-Nunes, J.M. Queiroz, G.O. Souza \& E.F. Ramos, 2007. Efeitos de fatores ambientais sobre a mirmecofauna em comunidade de restinga no Rio de Janeiro, RJ. Neotropical Entomology, 36: 28-37.

Yamaguchi, T. \& M. Hasegawa, 1996. Anti-predation mechanisms of soil animals against ants. Edaphologia, 30: 31-36.

Zar, J.H., 1999. Biostatistical analysis. New Jersey, PrenticeHall, 663 p.

Recebido em: 26/04/2010

Aceito em: 27/10/2010

$* * * * * * * * * * * * *$

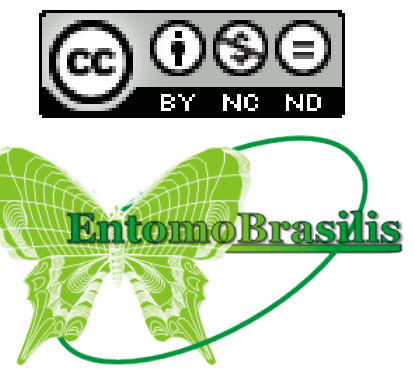

